\title{
Bulk properties of honeycomb lattices of superconducting microwave resonators
}

\author{
Alexis Morvan $\odot$, Mathieu Féchant, Gianluca Aiello, Julien Gabelli, and Jérôme Estève \\ Laboratoire de Physique des Solides, CNRS, Université Paris Saclay, Orsay, France
}

(Received 30 June 2021; revised 28 October 2021; accepted 22 November 2021; published 3 February 2022)

\begin{abstract}
We have designed different honeycomb lattices for microwave photons in the 4 to $8 \mathrm{GHz}$ band using superconducting spiral resonators. Each lattice comprises a few hundred sites. Two designs have been studied, one leading to two bands touching at the Dirac points and one where a gap opens at the Dirac points. Using a scanning laser technique to image the eigenmodes of these photonic lattices, we are able to reconstruct their band structure. The measured bands are in excellent agreement with ab initio models that combine numerical simulations of the electromagnetic properties of the spiral resonator and analytical calculations.
\end{abstract}

DOI: 10.1103/PhysRevResearch.4.013085

\section{INTRODUCTION}

Superconducting photonic lattices for microwave photons hold the promise to simulate the behavior of strongly interacting bosons in tailored $1 \mathrm{D}$ and 2D lattices [1-3]. In such systems, microwave photons with a typical frequency of a few $\mathrm{GHz}$ are stored in arrays of coupled superconducting resonators with a high-quality factor. The main interest of this platform is the possibility to reach the strongly interacting regime using Josephson junctions as a nonlinear element. But, Josephson junctions are prone to disorder, and the realization of large disorder free nonlinear lattices remains a difficult task. Nevertheless, many-body effects have already been demonstrated in small uni-dimensional lattices [4-8].

Independently of controlling the interactions in the lattice, experimental techniques must be developed to probe the properties of such large microwave circuits. In order to test these techniques, it seems reasonable to first characterize lattices in the linear regime and to quantitatively understand their properties $[9,10]$. This research direction, towards the realization of engineered band structures for photons, catches up with the rapidly growing field of photonic lattices and topological photonics [11-13]. In comparison to other photonic systems, the cryogenic environment and the low energy of the photons bring some technical difficulties.

In this paper, we use a laser scanning imaging technique in order to map the spatial distribution of the resonant modes that appear in lattices with a few hundred sites, where each site is a superconducting spiral resonator. This technique is similar to the laser scanning microscopy developed to observe the current density in superconductor films [14] and has already been used to characterize a single superconducting resonator [15,16] or coupled resonators [17]. It is an alternative to the probe scanning technique developed in [9]. We

Published by the American Physical Society under the terms of the Creative Commons Attribution 4.0 International license. Further distribution of this work must maintain attribution to the author(s) and the published article's title, journal citation, and DOI. apply this imaging method to three honeycomb lattices with different designs, where the A and B sites in the unit cell correspond to either identical or different resonators. From a Fourier analysis of the spatial distribution of the modes, we reconstruct the dispersion relation of the lattice bands. In the case where the A and B sites are identical, we observe two bands touching at the Dirac point, while in the other case, a gap opens at the Dirac points. These properties of the bands are similar to the ones for electrons in graphene and h-BN respectively. In order to understand our lattices and pinpoint the common features as well as the differences with electronic systems, we have developed two theoretical approaches to predict the properties of the lattice. The first approach consists in projecting the Maxwell equations on the basis of the resonator modes, which corresponds to a coupled mode theory (CMT), a technique widely used in photonics [18]. The band structure of the lattice is then obtained in terms of overlap integrals between the electric and magnetic fields of neighboring resonators. The second approach relies on more intensive numerical simulations to obtain an equivalent lumped element circuit to the lattice, from which the band structure can be calculated. These two ab initio approaches allow us to reproduce the measured band structures with good accuracy.

The paper is organized as follows. In the first part, we present the properties of the spiral resonator that is used as the site of the different lattices. In the second part, we present the measurement of the band structure of the different lattice designs. And the last part details the two models that we have developed to predict the band and mode structures of the lattices.

\section{SPIRAL RESONATOR PROPERTIES}

The three different lattices studied in this paper are honeycomb lattices, where each site consists of a superconducting spiral resonator as shown in Fig. 1. The spiral is made of a $4.3-\mu \mathrm{m}$ wide $\mathrm{Nb}$ wire that is winded in an hexagonal pattern with an overall size of $300 \mu \mathrm{m}$. The lattices are patterned through photo-lithography and reactive ion etching starting from a $\sim 300-n m$ thick sputtered $\mathrm{Nb}$ layer on top of a silicon wafer. The design of the spiral is adjusted through numerical 
(a)

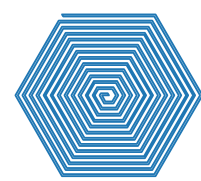

(b)

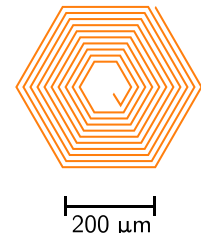

(c)

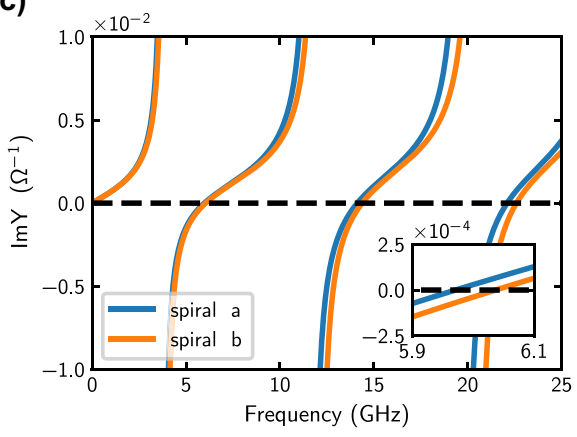

FIG. 1. [(a),(b)] Design of the two spirals used as the sites of our honeycomb lattices. The spiral shown in (a) consists of a $8.6 \mathrm{~mm}$ long wire with a width of $4.3 \mu \mathrm{m}$ and a gap between adjacent turns of $8.6 \mu \mathrm{m}$. The length of the (b) spiral is shorter resulting in a slightly higher resonance frequency. (c) Self-admittance of the two spirals calculated for a port located at the inner end of the wire with the Sonnet software [19]. The resonances correspond to the zero crossings. The inset shows a zoom close to the fundamental resonance around $6 \mathrm{GHz}$, the spacing between the two resonances is $120 \mathrm{MHz}$.

simulations with the Sonnet software [19], such that the resonance frequency $\omega_{0}$ of the fundamental mode is close to $2 \pi \times 6 \mathrm{GHz}$. This frequency can be finely tuned by adjusting the length of the wire at the center of the spiral. For the experiments shown in this paper, we use the two designs shown in Figs. 1(a) and 1(b) that are chosen to obtain two sites with slightly different $\omega_{0}$ spaced by $2 \pi \times 120 \mathrm{MHz}$. The Fig. 1(c) shows the simulated self-admittance of the two designs, from which we deduce $\omega_{0}$ as well as the resonance frequencies of the higher order modes. The second mode is expected to resonate around $14 \mathrm{GHz}$ and plays no role in the experiments presented here, where all measurements are performed between 5 and $7 \mathrm{GHz}$.

When two spirals are approached at a distance $d$ as shown in Fig. 2, the fundamental modes couple, giving rise to two resonances at frequencies $\omega_{-}$and $\omega_{+}$. If the coupling is not too strong, the coupled modes can be expressed as a linear combination of the uncoupled modes. This approach that consists in using a reduced set of well chosen modes as a basis is known as the coupled mode theory (CMT) [20-22]. Here, we use a basic CMT with only one mode per resonator. The Maxwell equations projected in this basis lead to a linear system, whose eigenvalues correspond to the resonance frequencies $\omega_{-}$and $\omega_{+}$. If the two resonators are identical with equal $\omega_{0}$, one obtains:

$$
\omega_{ \pm}=\sqrt{\frac{1 \pm \kappa_{e}}{1 \pm \kappa_{m}}} \omega_{0},
$$

where $\kappa_{e}\left(\kappa_{m}\right)$ is the electric (magnetic) coupling constant. These coupling constants are proportional to the overlap of the electric (magnetic) fields of the two resonators. We define

$$
\begin{aligned}
D_{i j} & =\int \epsilon(\mathbf{r}) \mathbf{E}_{i}(\mathbf{r}) \cdot \mathbf{E}_{j}(\mathbf{r}) d^{3} \mathbf{r}, \\
G_{i j} & =\int \mu_{0} \mathbf{H}_{i}(\mathbf{r}) \cdot \mathbf{H}_{j}(\mathbf{r}) d^{3} \mathbf{r},
\end{aligned}
$$
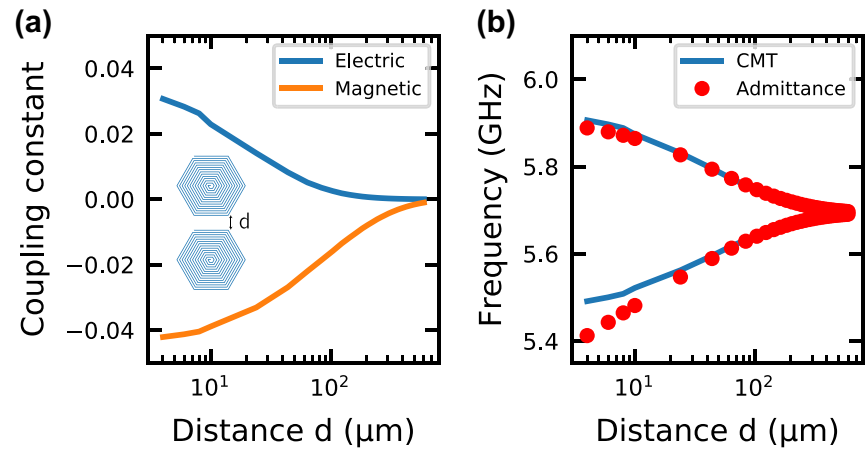

FIG. 2. Coupling between two spiral resonators as shown in Fig. 1(a). (a) Electric and magnetic coupling constants (see main text) as a function of $d$. (b) Resonance frequencies $\omega_{+}$and $\omega_{-}$of the two coupled resonators as a function of $d$. Red dots correspond to the result of a numerical simulation that computes the admittance matrix of the two coupled resonators using. The coupled mode theory prediction only uses the simulation of the electric and magnetic field created by a single resonator. It fails at short distances, where the resonator field is perturbed by the presence of the other resonator.

where $E_{i}\left(H_{i}\right)$ is the electric (magnetic) field of the mode associated with the $i=1,2$ resonator. We assume these functions to be real and explain in Appendix A how we compute the overlap integrals from the charge and current distribution in the spiral that is obtained from the numerical simulation of a single resonator. In particular, one has to take into account charge and current images due to the dielectric interface at the surface of the sample and to the metallic ground plane below the sample. The coupling constants are then given by $\kappa_{e}=D_{12} / D_{11}$ and $\kappa_{m}=G_{12} / G_{11}$. Figure 2(a) shows the evolution of $\kappa_{e}$ and $\kappa_{m}$ as a function of $d$ for two spirals shown in Fig. 1(a). Because $\kappa_{e}>0$ and $\kappa_{m}<0$, the magnetic and the electric couplings add up to increase the mode splitting, while the mean frequency $\left(\omega_{+}-\omega_{-}\right) / 2$ remains close to $\omega_{0}$. At large distances $(d>100 \mu \mathrm{m})$, the magnetic coupling dominates, while at short distances both couplings are important.

We compare the CMT predictions to a full numerical simulation of the two coupled resonators. From the simulation, we obtain the $2 \times 2$ admittance matrix $Y(\omega)$, which corresponds to the admittance matrix for two ports located at the inner ends of each spiral. The two resonances $\omega_{+}$and $\omega_{-}$are then obtained as the zeros of $\operatorname{det} Y(\omega)$. The CMT predictions coincide with the ones of the full numerical simulation at large distances $(d>20 \mu \mathrm{m})$ but fails at short distances as shown in Fig. 2(b). This is because, at short distances, the coupling is too strong and the coupled modes are not linear superposition of the uncoupled modes. In the following, we show results for lattices where $d=5 \mu \mathrm{m}$ or $d=30 \mu \mathrm{m}$. We therefore expect lattices with $d=30 \mu \mathrm{m}$ to be well described by the CMT method, while lattices with $d=5 \mu \mathrm{m}$ will require a more advanced model including the simulation of coupled sites. The half splitting $\left(\omega_{+}-\omega_{-}\right) / 2$ obtained from the admittance matrix method is $2 \pi \times 200 \mathrm{MHz}$ for $d=5 \mu \mathrm{m}$ and $2 \pi \times 120 \mathrm{MHz}$ for $d=30 \mu \mathrm{m}$. These values give an estimate of the nearest-neighbor coupling amplitude in a tight-binding description of the lattice. 

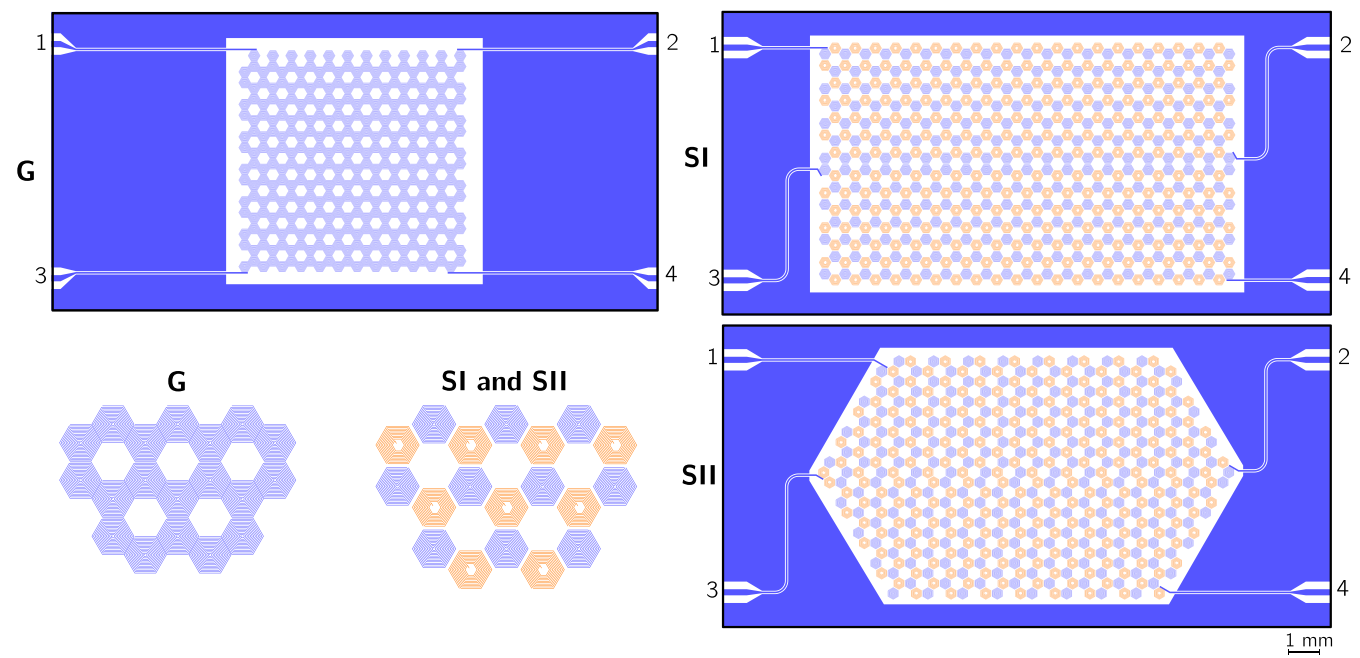

FIG. 3. Design of the three different honeycomb lattices studied in this article. Colored regions correspond to metal and white regions to the bare silicon. In the G design, all the sites are identical, while in the SI and SII designs, the A and B sites of the lattice correspond to two different resonators. The resonators correspond to the ones shown in Fig. 1 with the same coloring. Four coplanar waveguides, labeled 1 to 4 , are connected to single sites located at the edges of the lattice to probe the sample.

\section{LATTICE DESIGNS AND MEASUREMENTS}

Honeycomb lattices consisting of a few hundred spiral resonators are micro-fabricated on a $20 \times 10 \mathrm{~mm}^{2}$ sample, which is then mounted on the Still $(1 \mathrm{~K})$ stage of a dry dilution fridge. Three designs of honeycomb lattice labeled G, SI, and SII, (see Fig. 3) have been characterized. The distance between two site is denoted by $a$. The $\mathrm{G}$ design corresponds to the situation where all sites are identical, as in graphene, and are occupied by the spiral shown in Fig. 1(a). The other two designs, SI and SII, correspond to the more general situation where the two nonequivalent $\mathrm{A}$ and $\mathrm{B}$ sites of the honeycomb lattice are occupied by the two different spirals shown in Figs. 1(a) and 1(b). Because, to first approximation, the sites only differ by their on-site frequency, the SI and SII designs realize a so-called Semenoff insulator [23]. In the graphene case, the expected band structure consists of two bands touching at the two Dirac points, while in the Semenoff case a gap opens at the Dirac points. The value of the gap is given by the difference between the resonance frequencies of the A and B sites. In the two Semenoff designs, a horizontal boundary divides the sample in two halves: The A (B) sites in the lower half are occupied by the resonators that occupy the B (A) sites in the upper half. The two halves correspond to the same infinite lattice and therefore have the same bulk properties. The role of this boundary is to create valley Hall boundary states as discussed in [24].

\section{A. Transmission and density of states}

We probe the transmission through the lattice using four microwave ports that are connected to four coplanar waveguides. Each waveguide is coupled to a single site located on one edge of the lattice (see Fig. 3). We first characterize the bulk properties of the lattice by estimating the density of states (DOS) that we deduce from transmission measurements. Figure 4 shows a typical transmission spectrum obtained through a lattice of type SII. The lattice modes are visible as sharp resonant peaks. Depending on the sample, we identify between $43 \%$ (G design) and $80 \%$ (SI design) of the expected

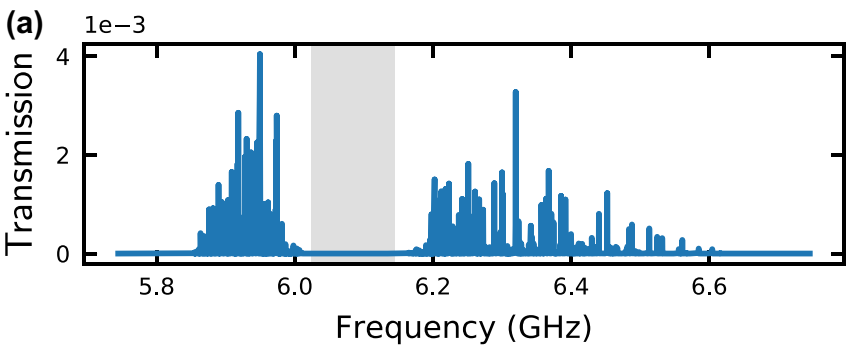

(b)

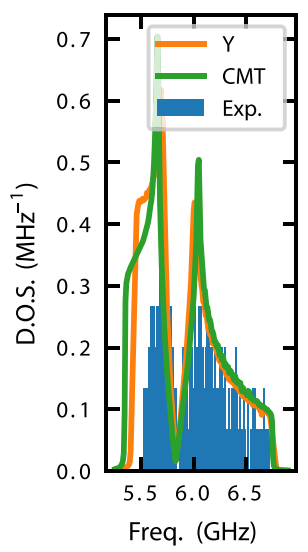

(c)

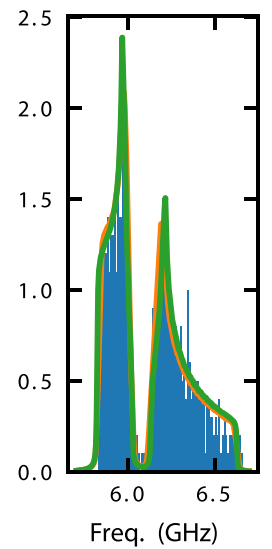

(d)

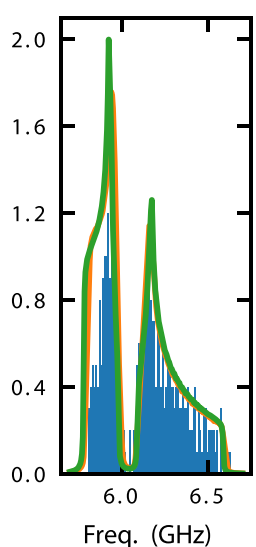

FIG. 4. (a) Transmission $\left|S_{41}\right|^{2}$ of the SI sample. The microwave setup used is detailed in Fig. S1 of the Supplemental Material [28]. The lattice modes appear as sharp resonant peaks. The transmission on resonance is below one indicating that the modes are under coupled: intrinsic loss dominate the coupling loss to the measurement waveguides. [(b),(c),(d)] The blue data show the estimated DOS from transmission spectra similar as the one shown in a for the three samples G, SI, and SII, respectively. The data are compared to the predictions of the two $a b$ initio models detailed in Sec. IV. The discrepancy with our data is due to the fact that we miss some peaks and underestimate the DOS. 
resonances. This number is limited by our signal to noise ratio and by the finite width of the peaks. Peaks that are too weakly coupled to the measurement ports are not identified as resonances and peaks too close in frequency are counted as a single peak. By counting the number of peaks in a frequency window of $15 \mathrm{MHz}$ for the design $\mathrm{G}$ and $10 \mathrm{MHz}$ for the design SI and SII, we can estimate the DOS as shown in Fig. 4. As expected, we observe two bands for all three samples, with a clear gap for the SI and SII samples. We can also identify the two van Hove singularities corresponding to the two maxima in the DOS of each band.

The two ab initio models to which we compare our data in the Fig. 4 are detailed in Sec. IV. The only adjustable parameter in the models is a global frequency offset. The frequencies of the remarkable points observed in the DOS (band minima, maxima and Dirac point) are well reproduced, but the measured DOS is not in quantitative agreement with the model predictions because a significant fraction of the modes is missing from the data.

\section{B. Mode imaging and dispersion relation}

In order to further characterize the lattice, we use a laser scanning technique to map the spatial variation of the modes identified in the transmission spectra. The measurement consists in monitoring the transmission of one (or many) mode while scanning a laser spot across the lattice. The optical setup (see Fig. 5) is designed to obtain a laser waist on the sample of $60 \mu \mathrm{m}$, which is much larger than the size of the spiral wire width and spacing, but smaller than the overall resonator size. This allows us to average the mode distribution over each site, while keeping sufficient resolution to resolve adjacent sites. We observe that the main effect of the laser is to induce dissipation on the illuminated site as observed in $[15,25]$. Because the modes are undercoupled to the probe ports, the loss increase induced by the laser results in a decrease of the mode transmission. To first order, the transmission drop for a given mode is proportional to the mode squared amplitude averaged over the illuminated area. In order to get rid of slow drifts and improve the signal to noise ratio, the laser intensity is modulated at a few $\mathrm{kHz}$. We then digitally demodulate the transmitted signal measured with a VNA and record the amplitude of the in phase signal as a function of the laser position.

Figure 5(d) shows a fine scan of one lattice site, which appears as a blurred hexagon with a central dip. This is consistent with the fact that the laser induced loss is maximal where the current density is large [25] as shown by the simulation in Fig. 5(e). We attribute a single value for the mode intensity per site by averaging over a few measurement points well inside the hexagon surrounding one site. We have checked that the final result is insensitive to the details of the averaging procedure. In order to optimize the acquisition time, different scanning techniques have been tested, continuous or raster. We obtain best results with a raster scan consisting of six measurement points per site, while we monitor the transmission change of all the peaks in a frequency window of about $1 \mathrm{GHz}$. This method allows us to image tens of modes in a single scan through the lattice.

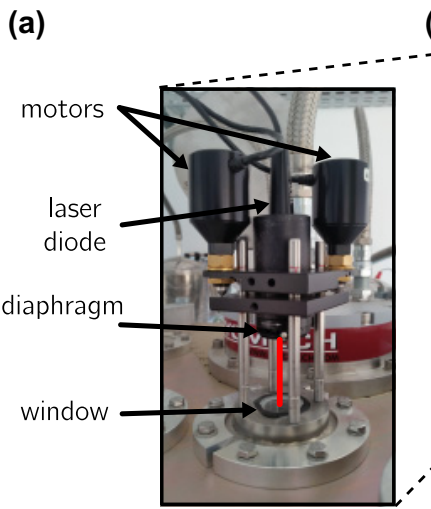

(b)

(c)

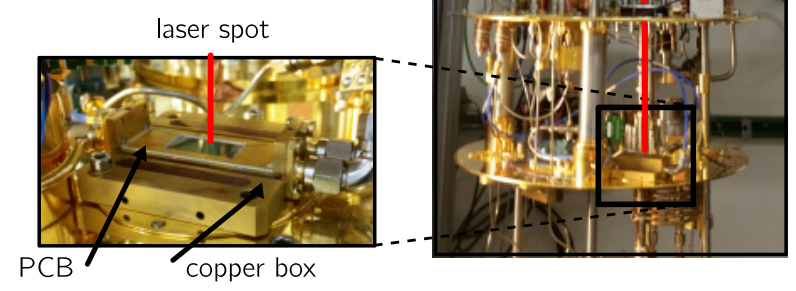

(d)

(e)
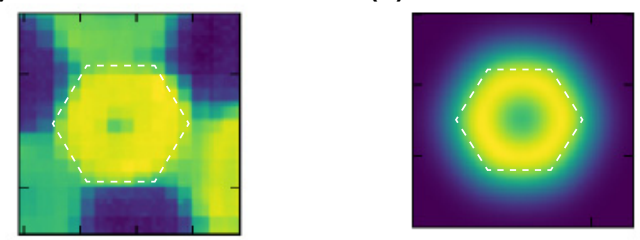

FIG. 5. [(a),(b),(c)] Laser scanning setup for mode imaging. A collimated laser beam is mounted on a motorized mirror mount outside the dry refrigerator (a). The beam is relayed by two lenses to pass through openings in the $50 \mathrm{~K}$ and the $4 \mathrm{~K}$ shields (b). A final lens focuses the beam onto the sample that is clamped on the $1 \mathrm{~K}$ stage (c). The tilt motion of the outer mirror mount results in a translation of the focused laser spot on the sample. [(d),(e)] Image of a single resonator in the lattice. The image in (d) shows the measured transmission of one resonant peak as a function of the laser position. The scanned area is centered around one site of the lattice. (e) The resulting image is well reproduced by the convolution of the simulated current intensity in the spiral with a Gaussian having a waist of $60 \mu \mathrm{m}$, as expected from the design of the optical setup.

Figures 6 and 7 show the results of the mode imaging technique applied to modes of the G, SI, and SII samples. In order to attribute a wave vector to each measured mode, we first use a sign reconstruction technique to deduce the signed mode amplitude from the measured intensity. The sign reconstruction is based on the assumption that the mode is a linear combination of $M$ known basis modes. As a basis, we take the modes of a lattice having the same geometry as the sample and assume a tight-binding model with nearestneighbor coupling only. We then look for the combination that matches best the measured mode intensity and use its sign to attribute a sign to the data. We have checked that the results are independent of $M$ when $M \geqslant 3$. Details of the method are given in Appendix B. In a second step, the Fourier transform of the reconstructed signed mode amplitude 
$6.8052 \mathrm{GHz}$

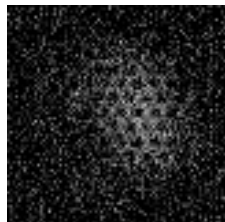

$6.7718 \mathrm{GHz}$

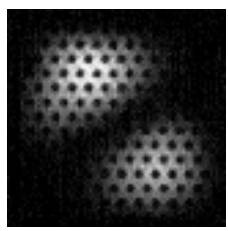

$6.6860 \mathrm{GHz}$

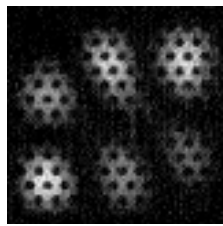

$6.6801 \mathrm{GHz}$

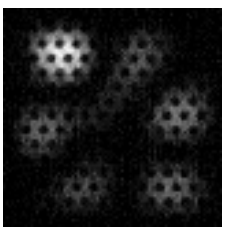

$6.6522 \mathrm{GHz}$

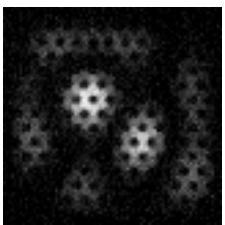

$6.6392 \mathrm{GHz}$

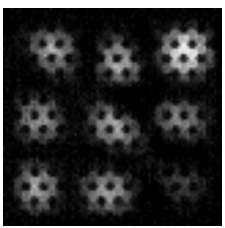

$6.6238 \mathrm{GHz}$
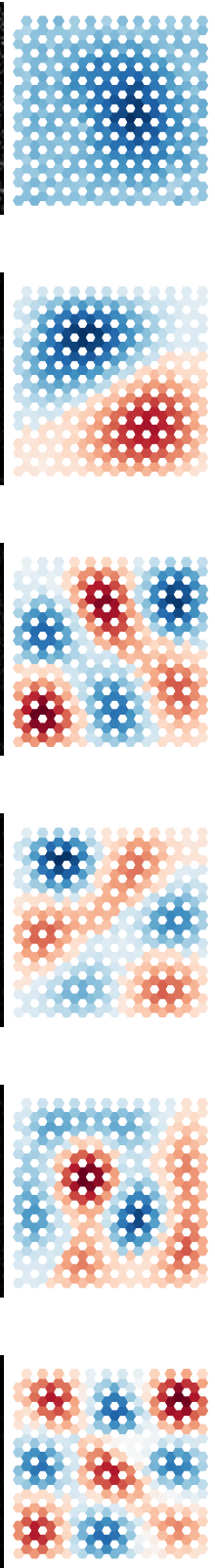

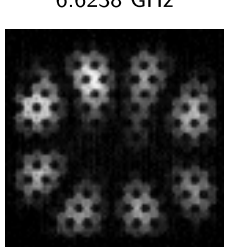

$6.6124 \mathrm{GHz}$

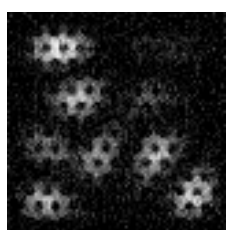

$6.5826 \mathrm{GHz}$

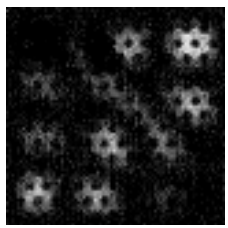

$6.5793 \mathrm{GHz}$

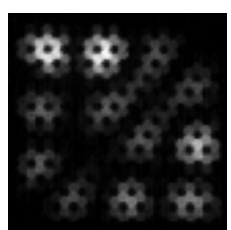

$6.5512 \mathrm{GHz}$

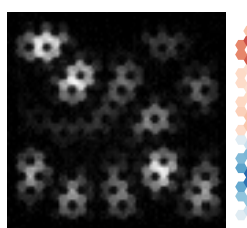

$6.5168 \mathrm{GHz}$

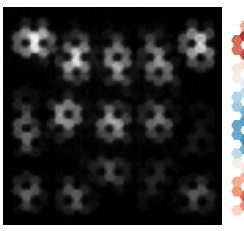

$6.4814 \mathrm{GHz}$

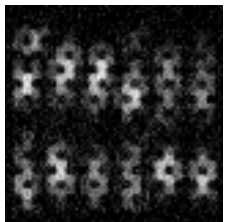

$6.4747 \mathrm{GHz}$
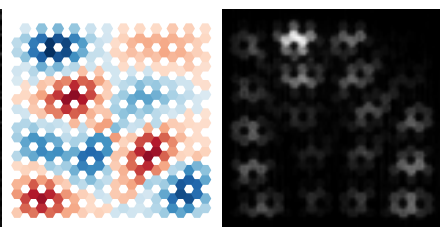

$6.4520 \mathrm{GHz}$
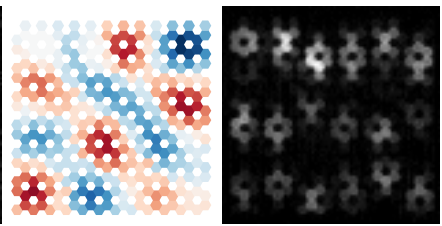

$6.4216 \mathrm{GHz}$
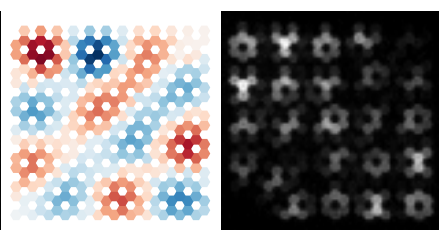

$6.4136 \mathrm{GHz}$

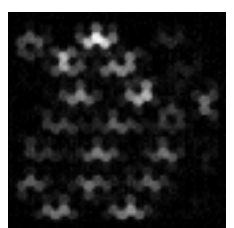

$6.4047 \mathrm{GHz}$

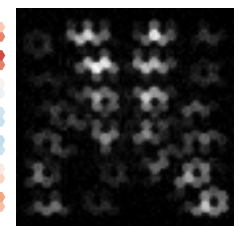

$6.3721 \mathrm{GHz}$
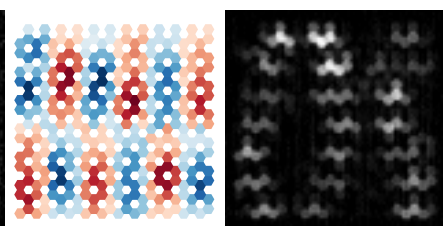

$6.3688 \mathrm{GHz}$

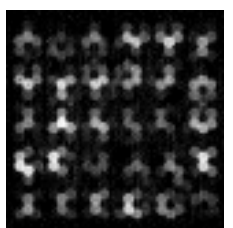

$\operatorname{sectst}$ $4 \times+2+1$

$3+1,4$ $460+8$ $2 \times 1 \times 00$

$6.3484 \mathrm{GHz}$
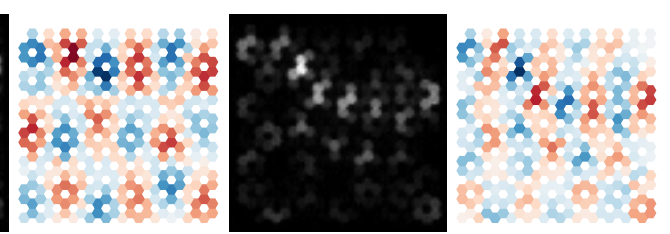

$6.3186 \mathrm{GHz}$
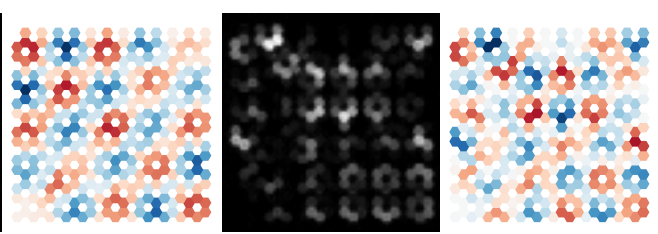

$6.3171 \mathrm{GHz}$
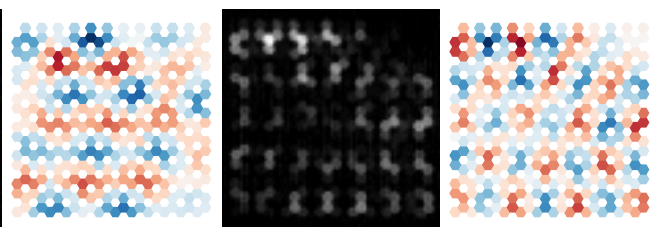

$6.2370 \mathrm{GHz}$
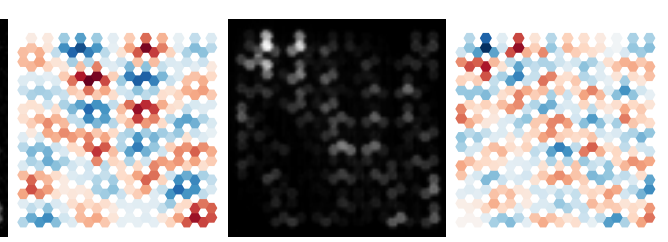

FIG. 6. Measured mode spatial dependence for the G sample. The black and white images show the raw data corresponding to the measured transmission change at the frequency of the mode indicated above each image. The grey scale is proportional to the mode intensity: white region correspond to large mode weight whereas dark region correspond to small mode weight. The corresponding red and blue image shows the reconstructed signed mode amplitude (see main text and Appendix B). Dark blue (red) indicates a large negative (positive) amplitude.

is calculated. In the reciprocal space, several peaks lying on a circle are observed. This allows us to attribute a single value $k$, corresponding to the norm of a wave vector, to each mode. Figure 8 shows the dispersion relation $\omega(k)$ that we obtain through this analysis for the G and SI samples. It corresponds to the radial projection of the $2 \mathrm{D}$ band structure. The measured dispersion for the SII sample is almost identical to the one of the SI sample and is not shown here. We recover the two asymmetric bands observed in the DOS estimation. The mode dispersion is quadratic at small $|k|$ leading to an effective mass for the photons in the lattice. At larger $k$, the dispersion relation clearly deviates from a quadratic behavior. For the $\mathrm{G}$ sample, we expect a linear dispersion around the Dirac points, but we are not able to image sufficiently many modes in this region to clearly reproduce this behavior. This is due to the finite size of the sample and also to the radial averaging over the direction of the wave vector. For the SI sample, we observe that the two bands curve in the opposite direction close to the Dirac points.

\section{LATTICE MODELS}

In comparison to the DOS, the measured dispersion shown in Fig. 8 is less affected by the fact that we are not able to probe all the modes. It allows us to precisely compare our data with two ab initio models. Following the analysis of the 


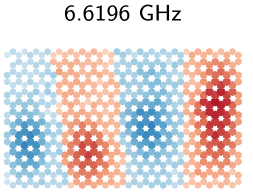

$6.5753 \mathrm{GHz}$

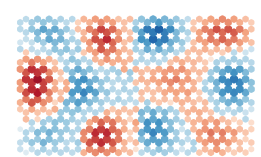

$6.5589 \mathrm{GHz}$

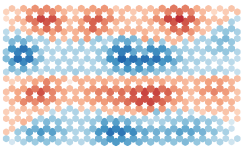

$6.5456 \mathrm{GHz}$

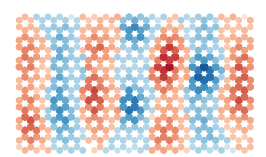

$6.4916 \mathrm{GHz}$

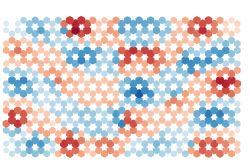

$6.6017 \mathrm{GHz}$

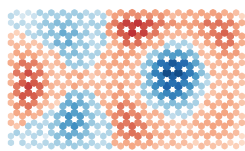

$6.5731 \mathrm{GHz}$

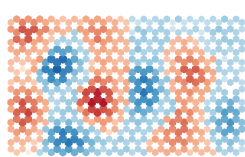

$6.5560 \mathrm{GHz}$

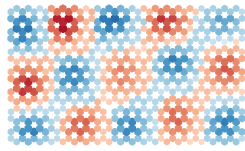

$6.5248 \mathrm{GHz}$

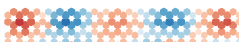

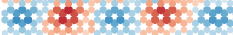

+WHA.t.6.

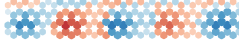

$6.4815 \mathrm{GHz}$

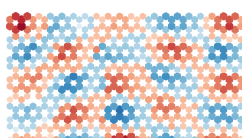

$6.5894 \mathrm{GHz}$

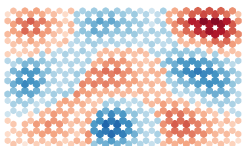

$6.5610 \mathrm{GHz}$

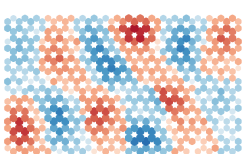

$6.5487 \mathrm{GHz}$

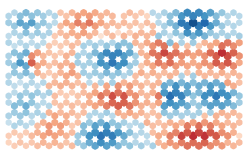

$6.5207 \mathrm{GHz}$

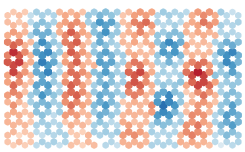

$6.4127 \mathrm{GHz}$
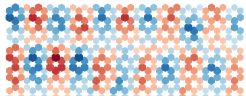

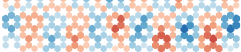

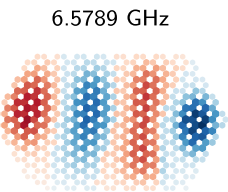

$6.4995 \mathrm{GHz}$

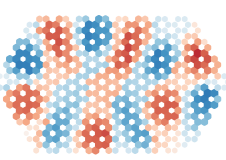

$6.4430 \mathrm{GHz}$

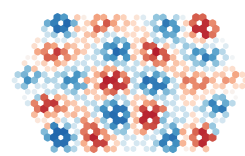

$6.3356 \mathrm{GHz}$

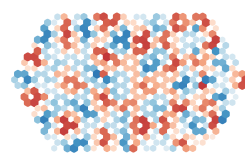

$6.3061 \mathrm{GHz}$

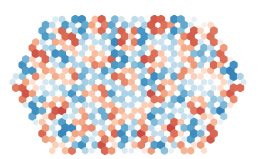

$6.5077 \mathrm{GHz}$

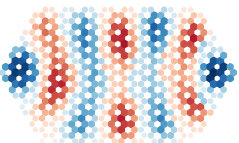

$6.4819 \mathrm{GHz}$

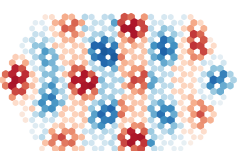

$6.4270 \mathrm{GHz}$

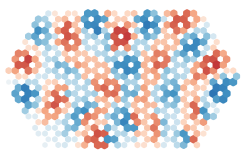

$6.3223 \mathrm{GHz}$

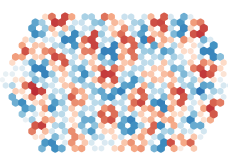

$6.2455 \mathrm{GHz}$

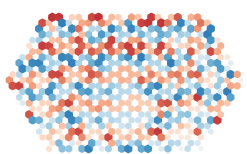

$6.5023 \mathrm{GHz}$

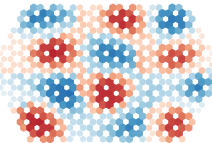

$6.4753 \mathrm{GHz}$

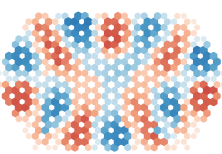

$6.3841 \mathrm{GHz}$

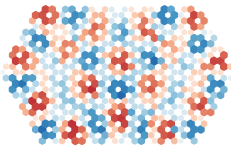

$6.3176 \mathrm{GHz}$

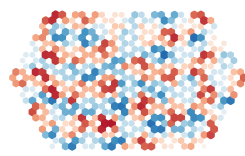

$6.2292 \mathrm{GHz}$

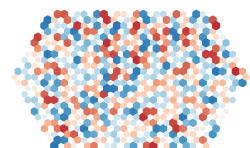

FIG. 7. Measured mode spatial dependence for the SI and SII samples. Here, we only show the reconstructed signed mode amplitudes (see Fig. 6). The frequency of the mode is indicated above each image.

coupling of two spiral resonators, we extend the CMT and the admittance matrix model to the case of an infinite lattice. The two models have no free parameters and differ in the following way: the CMT model solely relies on the simulation of the charge and current distribution of a single spiral at resonance, while the admittance matrix model simulates the admittance matrix of a cluster of a few coupled sites. As explained in Sec. II, the CMT model gives a clear physical picture of the coupling in the lattice in terms of overlap integrals between the different sites but fails when the coupling is too strong. As a consequence, the band structure of the SI or SII lattice is well predicted by the CMT but observe a discrepancy for the $\mathrm{G}$ lattice, which is more pronounced for the lower band. The $Y$ matrix calculation gives a more exact description of the lattice at any coupling but requires a more intensive numerical computation. In both models, we consider the coupling between one site and its nearest neighbours, up to the 3rd-nearest neighbours as shown in Fig. 9.

\section{A. Coupled mode theory}

We follow the derivation of [22] and adapt it to the specific case of coupled superconducting resonators. We only consider one mode per resonator and look for a solution to the Maxwell equations at a frequency $\omega$ as

$$
\begin{aligned}
\mathbf{E}(\mathbf{r}, t) & =\sum_{i} a_{i}(t) \mathbf{E}_{i}(\mathbf{r}), \\
\mathbf{H}(\mathbf{r}, t) & =\sum_{i} b_{i}(t) \mathbf{H}_{i}(\mathbf{r}),
\end{aligned}
$$

where $\mathbf{E}_{i}(\mathbf{r})\left[\mathbf{H}_{i}(\mathbf{r})\right]$ is the electric [magnetic] field of the mode associated to the resonator at site $i$. The two fields satisfy:

$$
\begin{aligned}
\nabla \times \mathbf{E}_{i} & =\mu_{0} \omega_{i} \mathbf{H}_{i}, \\
\nabla \times \mathbf{H}_{i} & =\epsilon_{0} \omega_{i} \mathbf{E}_{i}+\mathbf{J}_{i},
\end{aligned}
$$

where $\omega_{i}$ is the resonance frequency of the mode at site $i$ and $\mathbf{J}_{i}$ the associated current density. In addition to the overlap integrals defined in Eq. (3), we consider the integral

$$
M_{i j}=\int_{S}\left[\mathbf{E}_{i}(\mathbf{r}) \times \mathbf{H}_{j}(\mathbf{r})\right] \cdot d \mathbf{S},
$$

where the surface $S$ corresponds to all the metallic boundaries in the circuit, which consists of the resonators and the walls of the box enclosing the sample. This integral can be rewritten in terms of the overlap integrals $D_{i j}$ and $G_{i j}$ assuming that

$$
M_{i j} \approx \int_{S_{i} \cup S_{j}}\left[\mathbf{E}_{i}(\mathbf{r}) \times \mathbf{H}_{j}(\mathbf{r})\right] \cdot d \mathbf{S},
$$

where $S_{i}$ is the surface of the resonator at site $i$. The integral over $S_{i}$ is null because $\mathbf{E}_{i}(\mathbf{r})$ is normal to the surface of the resonator. Using that the current density in the resonator at site $j$ is given by $\mathbf{J}_{j}(\mathbf{r})=\mathbf{n} \times \mathbf{H}_{j}(\mathbf{r})$, where $\mathbf{n}$ is the normal to the resonator surface, we obtain

$$
M_{i j} \approx \int \mathbf{E}_{i}(\mathbf{r}) \cdot \mathbf{J}_{j}(\mathbf{r}) d^{3} \mathbf{r} .
$$

Using this approximation and considering the volume integral of $\nabla \cdot\left(\mathbf{E}_{i} \times \mathbf{H}_{j}\right)$, one obtains

$$
2 M_{i j}=\omega_{i} G_{i j}-\omega_{j} D_{i j} .
$$




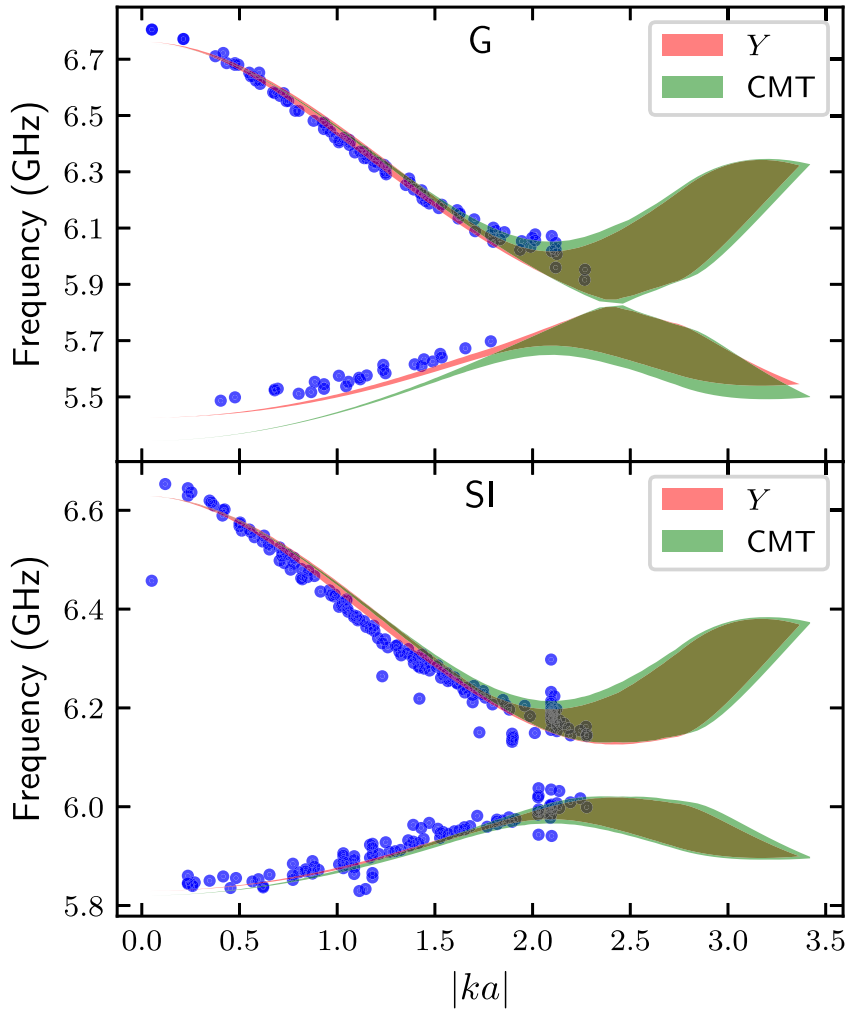

FIG. 8. Band structure of the G and SI samples. For a given resonance frequency, the corresponding norm $k$ of the wave vector associated to the mode is obtained from the Fourier analysis of the measured spatial dependence as shown in Figs. 6 and 7. The measured dispersion relation is compared to the predictions of the two ab initio models detailed in Sec. IV. The theoretical predictions are plotted as shaded areas, because, for a given $k$, the models predict different resonance frequencies depending on the orientation of the wave vector.

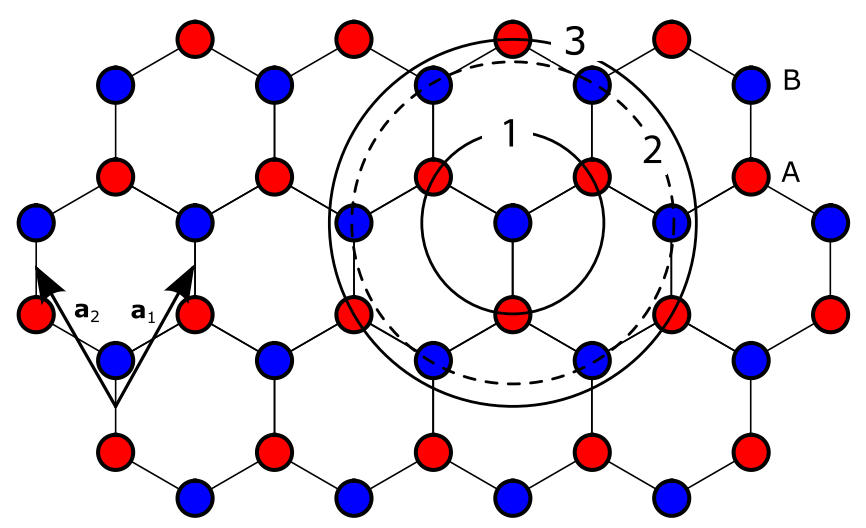

FIG. 9. We choose the primitive vectors of the lattice $a_{1}$ and $a_{2}$ as shown in the bottom-left corner. The different circles indicate the 1st, 2nd, and 3rd neighbor sites that we include in the calculation of the overlap of the electric and magnetic fields in the CMT model, or in the computation of the admittance matrix of the equivalent circuit describing the lattice.
In matrix form, the last equation writes

$$
2 M=\Omega G-D \Omega,
$$

where $\Omega$ is the diagonal matrix with elements $\Omega_{i i}=\omega_{i}$. In the same way, the volume integrals of $\nabla \cdot\left(\mathbf{H}_{i} \times \mathbf{E}\right)$ and $\nabla \cdot\left(\mathbf{E}_{i} \times\right.$ H) lead to the following two equations

$$
\begin{gathered}
G \dot{b}=-\Omega D a, \\
2 M b=\Omega G b-\omega D \dot{a} .
\end{gathered}
$$

Using the approximation from Eq. (4) for $M$ and eliminating $b$, we look for periodic solutions at frequency $\omega$ and obtain the following eigenvalue problem

$$
\omega^{2} a=\Omega G^{-1} \Omega D a .
$$

In the case of two resonators only, we recover the result given in Eq. (1). The matrices $G, \Omega$, and $D$ are real symmetric matrices, but, in general, $\Omega G^{-1} \Omega D$ is not symmetric. Its eigenvalues are real and positive with, in general, nonorthogonal eigenvectors.

Equation (7) can be used to find the coupled mode frequencies of any ensemble of coupled resonators. In order to take advantage of the lattice periodicity, we relabel the $a_{i}$ and $b_{i}$ amplitudes in a given lattice cell as $a_{\mu}(\mathbf{R})$ and $b_{\mu}(\mathbf{R})$, where $\mathbf{R}$ is the Bravais lattice vector identifying the position of the cell in the lattice and the $\mu$ index distinguishes the $\mathrm{A}$ from the B sites. Using the same notation, we define the following overlap integrals between two sites spaced by $\mathbf{R}$ in the lattice

$$
\begin{aligned}
\left\langle\mathbf{E}_{\mu} \mathbf{E}_{v}\right\rangle_{\mathbf{R}} & =\int \epsilon(\mathbf{r}) \mathbf{E}_{\mu}(r) \cdot \mathbf{E}_{v}(\mathbf{r}-\mathbf{R}) d^{3} \mathbf{r}, \\
\left\langle\mathbf{H}_{\mu} \mathbf{H}_{v}\right\rangle_{\mathbf{R}} & =\int \mu_{0} \mathbf{H}_{\mu}(\mathbf{r}) \cdot \mathbf{H}_{v}(\mathbf{r}-\mathbf{R}) d^{3} \mathbf{r} .
\end{aligned}
$$

We now look for periodic solutions over the lattice as

$$
a_{\mu}(R)=e^{i \mathbf{k} \cdot \mathbf{R}} a_{\mu}(\mathbf{k}) b_{\mu}(R)=e^{i \mathbf{k} \cdot \mathbf{R}} b_{\mu}(\mathbf{k}),
$$

and define the Fourier transform of the $D$ and $G$ overlap matrices

$$
\begin{aligned}
& D_{\mu \nu}(\mathbf{k})=\sum_{\mathbf{R}} e^{i \mathbf{k} \cdot \mathbf{R}}\left\langle\mathbf{E}_{\mu} \mathbf{E}_{\nu}\right\rangle_{\mathbf{R}}, \\
& G_{\mu \nu}(\mathbf{k})=\sum_{\mathbf{R}} e^{i \mathbf{k} \cdot \mathbf{R}}\left\langle\mathbf{H}_{\mu} \mathbf{H}_{\nu}\right\rangle_{\mathbf{R}} .
\end{aligned}
$$

We suppose that the lattice contains $N$ cells and we look for solutions with periodic boundary conditions such that $\sum_{\mathbf{R}} e^{i \mathbf{k} \cdot \mathbf{R}}=N \delta_{\mathbf{k}, 0}$, where $\mathbf{k}$ can take $N$ values in the first Brillouin zone. Equations (5) and (6) then lead to

$$
\Omega G^{-1}(\mathbf{k}) \Omega D(\mathbf{k}) a(\mathbf{k})=\omega^{2}(\mathbf{k}) a(\mathbf{k}),
$$

where $\Omega$ is now the $2 \times 2$ diagonal matrix with the resonance frequencies of the $\mathrm{A}$ and $\mathrm{B}$ sites. The resulting eigenvalue problem in Eq. (10) gives the dispersion of the two bands as a function of $\mathbf{k}$. We compute the band structure by including all couplings up to the third-neighbor coupling (see Fig. 9) in Eqs. (8) and (9). The results are shown in Fig. 8.

In order to compare the photonic band structure with the one of a tight-binding model with the same range of coupling, we consider the case of the $\mathrm{G}$ lattice where the A and B sites have the same resonance frequency. In order to obtain a simple 
analytical formula, we neglect the dependence of the overlap integrals with the direction of separation, e.g., we suppose that $\left\langle\mathbf{E}_{B} \mathbf{E}_{A}\right\rangle_{0}=\left\langle\mathbf{E}_{B} \mathbf{E}_{A}\right\rangle_{\mathbf{a}_{1}}=\left\langle\mathbf{E}_{B} \mathbf{E}_{A}\right\rangle_{\mathbf{a}_{2}}$. With this approximation, a straightforward calculation gives that $D(\mathbf{k})$ and $G(\mathbf{k})$ are diagonal in the same basis, leading to the following dispersion relation $\omega_{ \pm}(\mathbf{k})$ :

$$
\omega_{ \pm}(\mathbf{k})=\sqrt{\frac{1+\kappa_{e}^{(2)} f_{2}(\mathbf{k}) \pm\left|\kappa_{e}^{(1)} f_{1}(\mathbf{k})+\kappa_{e}^{(3)} f_{3}(\mathbf{k})\right|}{1+\kappa_{m}^{(2)} f_{2}(\mathbf{k}) \pm\left|\kappa_{m}^{(1)} f_{1}(\mathbf{k})+\kappa_{m}^{(3)} f_{3}(\mathbf{k})\right|}} \omega_{0},
$$

where the electric coupling constants are given by

$$
\begin{aligned}
\kappa_{e}^{(1)} & =\left\langle\mathbf{E}_{A} \mathbf{E}_{B}\right\rangle_{\mathbf{0}} /\left\langle\mathbf{E}_{A} \mathbf{E}_{A}\right\rangle_{\mathbf{0}}, \\
\kappa_{e}^{(2)} & =\left\langle\mathbf{E}_{A} \mathbf{E}_{A}\right\rangle_{\mathbf{a}_{1}} /\left\langle\mathbf{E}_{A} \mathbf{E}_{A}\right\rangle_{\mathbf{0}}, \\
\kappa_{e}^{(3)} & =\left\langle\mathbf{E}_{A} \mathbf{E}_{A}\right\rangle_{\mathbf{a}_{1}+\mathbf{a}_{2}} /\left\langle\mathbf{E}_{A} \mathbf{E}_{A}\right\rangle_{\mathbf{0}} .
\end{aligned}
$$

The magnetic couplings $\kappa_{m}^{(i)}$ are given by the same expressions in terms of magnetic field overlaps and the link functions $f_{i}(\mathbf{k})$ correspond to

$$
\begin{aligned}
& f_{1}(\mathbf{k})=1+e^{i \mathbf{k} \cdot \mathbf{a}_{1}}+e^{i \mathbf{k} \cdot \mathbf{a}_{2}}, \\
& f_{2}(\mathbf{k})=2\left[\cos \mathbf{k} \cdot \mathbf{a}_{1}+\cos \mathbf{k} \cdot \mathbf{a}_{2}+\cos \mathbf{k} \cdot\left(\mathbf{a}_{1}-\mathbf{a}_{2}\right)\right], \\
& f_{3}(\mathbf{k})=e^{i \mathbf{k} \cdot\left(\mathbf{a}_{1}+\mathbf{a}_{2}\right)}+2 \cos \mathbf{k} \cdot\left(\mathbf{a}_{1}-\mathbf{a}_{2}\right) .
\end{aligned}
$$

Expression (11) can be compared to the tight-binding dispersion relation

$$
\omega_{ \pm}^{\mathrm{TB}}(\mathbf{k})=\omega_{0}+t_{2} f_{2}(\mathbf{k}) \pm\left|t_{1} f_{1}(\mathbf{k})+t_{3} f_{3}(\mathbf{k})\right|,
$$

which comes from the diagonalization of the tight-binding Hamiltonian

$$
H^{\mathrm{TB}}(\mathbf{k})=\left(\begin{array}{cc}
\omega_{0}+t_{2} f_{2}(\mathbf{k}) & t_{1} f_{1}(\mathbf{k})+t_{3} f_{3}(\mathbf{k}) \\
t_{1} f_{1}^{*}(\mathbf{k})+t_{3} f_{3}^{*}(\mathbf{k}) & \omega_{0}+t_{2} f_{2}(\mathbf{k})
\end{array}\right) .
$$

The coupling $t_{i}$ corresponds to the $i$ th nearest-neighbor coupling. The dispersion relation equations (11) and (12) match if one keeps only nearest-neighbor terms, performs a first-order expansion in $\kappa_{e}^{(1)}$ and $\kappa_{m}^{(1)}$ and identifies $t_{1}$ to $\omega_{0}\left(\kappa_{e, 1}-\kappa_{m, 1}\right) / 2$. Including 2nd and 3rd nearest-neighbor overlap integrals lead to a dispersion relation that cannot be identified to its tight-binding counterpart with the same coupling range.

As mentioned in the introduction, a strong motivation to build lattices of superconducting resonators is to include nonlinear elements in the lattice. For example, in the case considered here, one could design spirals with an empty area in the center, where a transmon might be inserted and coupled each site. In this context, it is interesting to obtain the Hamiltonian that describes the linear behavior of the lattice as a starting point to write the non-linear Hamiltonian. Using the CMT approach, we obtained the two equations of motion

$$
\dot{a}=\Omega b, \quad G \dot{b}=-\Omega D a .
$$

And the total energy in the system is given by

$$
H=\frac{1}{2} a^{T} D a+\frac{1}{2} b^{T} G b .
$$

In order to identify $a$ and $b$ with the two quadratures of the resonator modes, we normalize the basis fields to

$$
D_{i i}=G_{i i}=\hbar \omega_{i} \text {. }
$$

With this definition, and in the absence of coupling between the resonators $(G=D=\hbar \Omega)$, the equations of motion coincide with the Hamilton equations derived from $H$ assuming standard commutation rules between the mode quadratures, $\left[a_{m}, a_{n}\right]=0,\left[b_{m}, b_{n}\right]=0$ and $\left[a_{m}, b_{n}\right]=i \hbar \delta_{m n}$. In the following, we drop the $\hbar$ factors and set $\hbar=1$. When the overlap between adjacent sites are nonzero, the equations of motion do not coincide anymore with the naive Hamilton equations obtained by considering that the $a$ 's and $b$ 's are conjugate variables. This comes from the fact that the basis used to project the Maxwell equations is not orthonormal, which modifies the commutation relations. This problem is well known in the calculation of electronic band structure using the LCAO method. In order to find an orthonormal basis, we adapt the Löwdin procedure used for electrons [26]. We first apply the canonical transformation $a \rightarrow \Omega^{1 / 2} a$ and $b \rightarrow \Omega^{-1 / 2} b$ to remove the $\Omega$ dependence in the equation of motion, which transforms the Hamiltonian to

$$
H=\frac{1}{2} a^{T} \tilde{D} a+\frac{1}{2} b^{T} \tilde{G} b,
$$

with $\tilde{D}=\Omega^{1 / 2} D \Omega^{1 / 2}$ and $\tilde{G}=\Omega^{-1 / 2} G \Omega^{-1 / 2}$. The equations of motion become $\dot{a}=b$ and $G \dot{b}=-D a$. We now apply the Löwdin transformation $a \rightarrow \tilde{G}^{-1 / 2} a$ and $b \rightarrow \tilde{G}^{-1 / 2} b$ in order to restore standard commutation relations. The Hamiltonian becomes

$$
H=\frac{1}{2} a^{T} \tilde{G}^{-1 / 2} \tilde{D} \tilde{G}^{-1 / 2} a+\frac{1}{2} b^{T} b .
$$

The equations of motion are modified to $\dot{a}=b$ and $\dot{b}=$ $-\tilde{G}^{-1 / 2} \tilde{D} \tilde{G}^{-1 / 2} a$, which now correspond to the standard Hamilton equations. We finally apply the canonical transformation $a \rightarrow \Omega^{-1 / 2} a$ and $b \rightarrow \Omega^{1 / 2} b$, which reverses the effect of the first transformation in order to explicitly restore the $\Omega$ dependence. The final Hamiltonian is

$$
H=\frac{1}{2} a^{T} \Omega^{-1 / 2} \tilde{G}^{-1 / 2} \tilde{D} \tilde{G}^{-1 / 2} \Omega^{-1 / 2} a+\frac{1}{2} b^{T} \Omega b .
$$

We can now introduce the bosonic operators $c=(a+$ ib) $/ \sqrt{2}$, which fulfill $\left[c_{m}, c_{n}\right]=0$ and $\left[c_{m}, c_{n}^{\dagger}\right]=\delta_{m n}$. The Hamiltonian $H$ can be rewritten as

$$
H=\frac{1}{2}\left(c^{\dagger} \Omega c+c \Omega c^{\dagger}\right)+\frac{1}{2}\left(c+c^{\dagger}\right)^{T} J\left(c+c^{\dagger}\right),
$$

with $2 J=\Omega^{-1 / 2} \tilde{G}^{-1 / 2} \tilde{D} \tilde{G}^{-1 / 2} \Omega^{-1 / 2}-\Omega$. A rotating wave approximation (RWA) can then be performed to transform the last term to $c^{T}(J / 2) c^{\dagger}+$ H.c. By construction, the normal mode frequencies of the Hamiltonian Eq. (13) are the identical to the ones obtained with the CMT. The RWA introduces a negligible error, on the order of $0.1 \%$ for the SI or SII lattice.

In order to compute the matrix $J$ for an infinite lattice, we start from the expressions of $D(\mathbf{k})$ and $G(\mathbf{k})$ in the Fourier space defined in Eqs. (8) and (9). The expression of $J$ between two lattice cells separated by a vector $\mathbf{R}$ is then given by

$$
\begin{aligned}
J(\mathbf{R})= & -\frac{\Omega}{2} \delta_{\mathbf{R}, 0}+\frac{1}{2 N_{s}} \\
& \times \sum_{\mathbf{k}} e^{-i \mathbf{k} \cdot \mathbf{R}} \Omega^{-1 / 2} \tilde{G}(\mathbf{k})^{-1 / 2} \tilde{D}(\mathbf{k}) \tilde{G}(\mathbf{k})^{-1 / 2} \Omega^{-1 / 2},
\end{aligned}
$$

where the $\tilde{D}(\mathbf{k})$ and $\tilde{G}(\mathbf{k})$ matrices are defined as

$$
\begin{aligned}
\tilde{G} & =\Omega^{-1 / 2} G(\mathbf{k}) \Omega^{-1 / 2}, \\
\tilde{D} & =\Omega^{1 / 2} D(\mathbf{k}) \Omega^{1 / 2} .
\end{aligned}
$$


The values of $J(\mathbf{R})$ for the SI and SII samples are given in the Appendix $\mathrm{C}$. We obtain an average nearest-neighbour coupling of $2 \pi \times 124 \mathrm{MHz}$ and a, next nearest-neighbour coupling of $2 \pi \times 18 \mathrm{MHz}$. Keeping only the NN coupling in the computation of $D(\mathbf{k})$ and $G(\mathbf{k})$, we obtain a small, $2 \pi \times 4 \mathrm{MHz}$, but nonzero value as a result of the Löwdin transform.

\section{B. Admittance matrix}

In order to model more accurately the G lattice, we extend the admittance matrix method introduced in Sec. II to an infinite lattice of resonators. We model the lattice by defining $M \geqslant 1$ microwave ports inside each resonator, and introduce the set of $N$ complex vectors $V(\mathbf{R})$, each with $2 M$ components, which correspond to the voltages at the $2 M$ ports in the lattice cell at position $\mathbf{R}$. At a given frequency $\omega$, the Kirchhoff's circuit laws describing the lattice can be written

$$
\sum_{\mathbf{R}} Y_{\mathbf{R}}(\omega) V(\mathbf{R})=0
$$

where the sum over $\mathbf{R}$ is over the points of the Bravais lattice. The matrix $Y_{0}(\omega)$ corresponds to the admittance matrix between the ports belonging to the same cell, while $Y_{\mathbf{R}}(\omega)$ is the admittance matrix between the ports in two cells separated by $\mathbf{R}$. The admittance matrices satisfy $Y_{\mathbf{R}}(\omega)=Y_{\mathbf{R}}^{T}(\omega)$. We look for a periodic solution $V(\mathbf{R})=V(\mathbf{k}) e^{i \mathbf{k R}}$ and obtain

$$
\sum_{\mathbf{R}} Y_{\mathbf{R}}(\omega) e^{i \mathbf{R} \mathbf{R}} V(\mathbf{k})=0,
$$

where $V(\mathbf{k})$ is a $2 M$ element vector. The dispersion relation is then obtained by numerically solving

$$
\operatorname{det}\left(\sum_{\mathbf{R}} Y_{\mathbf{R}}(\omega) e^{i \mathbf{k} \mathbf{R}}\right)=0,
$$

for $\omega$ at a given $\mathbf{k}$. The $Y_{\mathbf{R}}(\omega)$ matrix is obtained from the numerical simulation of a small lattice flake. The CMT calculations indicate that one should simulate a flake with enough sites such that the central site is surrounded by at least its 3rd nearest neighbours. We therefore choose the geometry shown in Fig. 10(b) with 16 sites. In order for the sum over $\mathbf{R}$ to converge rapidly, when the distance between sites increases, the number of ports $M$ and their location on the spiral must be well chosen. We observe that $M=1$ is not sufficient, while results obtained for $M>2$ and various port locations give the same results. The results of the final simulations shown in Fig. 8 are obtained with $M=3$ ports located as shown in Fig. 10(a).

Compared to the CMT model, the admittance matrix is numerically more demanding because it requires the simulation of a much larger number of sites. The dispersion obtained for the SI and SII lattice confirms the prediction of the CMT model, which were already in good agreement with our experimental data. For the $\mathrm{G}$ lattice, we observe a deviation compared to the CMT model and a better agreement with the measured dispersion. (a)

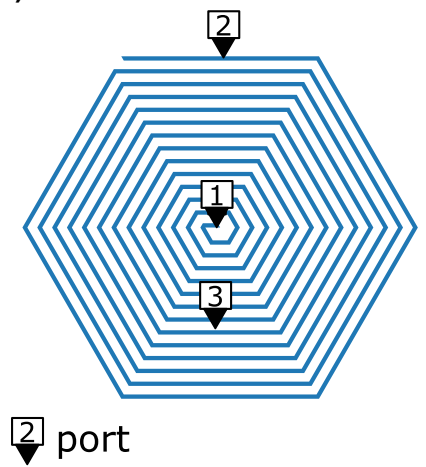

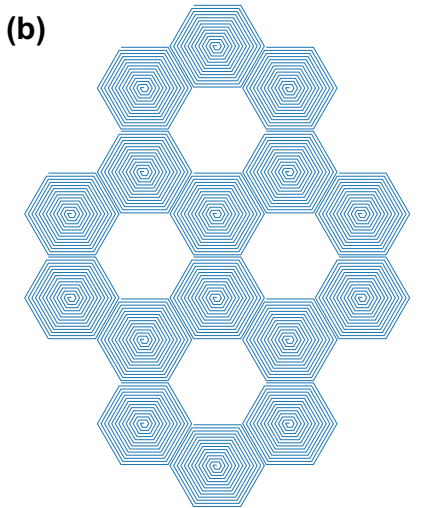

FIG. 10. (a) Location of the ports used to simulate the admittance matrix describing the lattice. We use the Sonnet software in order to obtain the admittance between all the ports of the small lattice shown in b). The lattice flake is chosen so that the admittance between one of the central site and its 1st, 2nd, and 3rd nearest neighbours can be extracted from the simulation.

\section{CONCLUSION}

In conclusion, we have shown that a laser scanning technique may be used to image the modes that appear in relatively large lattices of superconducting resonators. Here, the technique was applied to niobium resonators at a temperature around $1 \mathrm{~K}$, but we believe that the same method could be extended to characterize lattices or networks of superconducting resonators at lower temperatures that may include Josephson junctions. The mode images allowed us to reconstruct the radial projection of the dispersion relation in the lattice. The two different models that we present accurately reproduce the measured dispersion. In particular, we show how to obtain a tight-binding description from the knowledge of the electric and magnetic couplings between the lattice sites. Such models and their experimental validation are one of the prerequisite to the simulation of interacting lattices in circuit QED systems.

\section{ACKNOWLEDGMENTS}

The authors gratefully acknowledge the insights of $\mathrm{M}$. Aprili, G. Montambaux, J.-N. Fuchs, and F. Piechon. This work is supported by the Agence Nationale de la Recherche (ANR-18-CE47-0003 BOCA project) and the Laboratoire d'excellence Physique Atomes Lumière Matière (ANR-10LABX-0039-PALM).

\section{APPENDIX A: OVERLAP INTEGRALS}

The overlap integral of the electric fields associated to the $i$ and $j$ sites, as defined in Eq. (2), can be rewritten in terms of the charge distribution $\rho_{i}$ of the $i$ site and the electric potential $\phi_{j}$ of the $j$ site [27]. Similarly, the overlap between the magnetic fields in Eq. (3) can be rewritten in terms of the current distribution $\mathbf{j}_{i}$ and vector potential $\mathbf{A}_{j}$. One obtains

$$
\begin{aligned}
& E_{E}=\int \epsilon(\mathbf{r}) \mathbf{E}_{i}(\mathbf{r}) \cdot \mathbf{E}_{j}(\mathbf{r}) d^{3} \mathbf{r}=\int \rho_{i}(\mathbf{r}) \phi_{j}(\mathbf{r}) d^{3} \mathbf{r} \\
& E_{H}=\int \mu_{0} \mathbf{H}_{i}(\mathbf{r}) \cdot \mathbf{H}_{j}(\mathbf{r}) d^{3} \mathbf{r}=\int \mathbf{j}_{i}(\mathbf{r}) \cdot \mathbf{A}_{j}(\mathbf{r}) d^{3} \mathbf{r} .
\end{aligned}
$$


In order to compute $\phi_{j}$ and $\mathbf{A}_{j}$ from $\rho_{j}$ and $\mathbf{j}_{j}$, the presence of the dielectric substrate and of the metallic ground plane located below the substrate must be taken into account using image charges and currents. We obtain

$$
E_{E}=\frac{1-k}{4 \pi \epsilon_{0}} \int \rho_{i}(\mathbf{r}) \rho_{j}\left(\mathbf{r}^{\prime}\right) f\left(\mathbf{r}, \mathbf{r}^{\prime}\right) d^{3} \mathbf{r} d^{3} \mathbf{r}^{\prime}
$$

with $f\left(\mathbf{r}, \mathbf{r}^{\prime}\right)=\left(\frac{1}{\left|\mathbf{r}-\mathbf{r}^{\prime}\right|}+\sum_{n=1}^{M} \frac{(-1)^{n} k^{n-1}(1+k)}{\left|\mathbf{r}-\mathbf{r}^{\prime}+2 n e \mathbf{z}\right|}\right)$

$$
E_{H}=\frac{\mu_{0}}{4 \pi} \int \mathbf{j}_{i}(\mathbf{r}) \cdot \mathbf{j}_{j}\left(\mathbf{r}^{\prime}\right) g\left(\mathbf{r}, \mathbf{r}^{\prime}\right) d^{3} \mathbf{r} d^{3} \mathbf{r}^{\prime}
$$

with $g\left(\mathbf{r}, \mathbf{r}^{\prime}\right)=\left(\frac{1}{\left|\mathbf{r}-\mathbf{r}^{\prime}\right|}-\frac{1}{\left|\mathbf{r}-\mathbf{r}^{\prime}+2 e \mathbf{z}\right|}\right)$

where $e$ is the wafer thickness, $\mathbf{z}$ is the unit vector normal to the substrate, $k=\left(\epsilon_{r}-1\right) /\left(\epsilon_{r}+1\right), \epsilon_{r} \approx 11.8$ is the dielectric constant of the silicon substrate, and $M$ is the number of image charges at which the sum is truncated. We use $M=4$ for the simulations shown in the article. These expressions neglect any propagation effect, which is well justified in our situation. We numerically compute the last two integrals from the knowledge of the charge and current distributions $\rho_{i}$ and $\mathbf{j}_{i}$ that we obtain from the Sonnet simulation software.

\section{APPENDIX B: SIGN RECONSTRUCTION OF THE MODE AMPLITUDE}

From the variation of the transmission at resonance as a function of the laser position, we obtain the mode intensity $|\Psi(\mathbf{r})|^{2}$, where $\mathbf{r}$ takes $N$ discrete values over the sites of the lattice. The mode is normalized such that $\sum_{\mathbf{r}}|\Psi(\mathbf{r})|^{2}=1$. In order to obtain $\Psi(\mathbf{r})$, we assume that the mode is a linear combination of the modes that are expected for a lattice with the same geometry as the measured one and nearest-neighbor coupling only. In the case of the $G$ lattice, the basis modes $\phi_{\alpha}(\mathbf{r})$ only depends on the lattice geometry. In the case of the SI or SII lattice, the modes also depend on the ratio of the nearest-neighbor hopping to the frequency imbalance between the A and B sites. We set this ratio to the one obtained from simulations of two coupled resonators.

We then search for the unit vector $\beta$ such that

$$
L(\beta)=\sum_{\mathbf{r}}\left(|\Psi(\mathbf{r})|^{2}-\left|\sum_{\alpha=1}^{M} \beta_{\alpha} \phi_{\alpha}(\mathbf{r})\right|^{2}\right)^{2}
$$

is minimal, where $M$ is the number of modes used to reconstruct $\Psi(\mathbf{r})$. The quantity $L(\beta)$ can be rewritten

$$
\begin{aligned}
L(\beta)= & \sum_{\mathbf{r}}\left|\sum_{\alpha=1}^{M} \beta_{\alpha} \phi_{\alpha}(\mathbf{r})\right|^{4}-2 \sum_{\mathbf{r}}|\Psi(\mathbf{r})|^{2}\left|\sum_{\alpha=1}^{M} \beta_{\alpha} \phi_{\alpha}(\mathbf{r})\right|^{2} \\
& +\sum_{\mathbf{r}}|\Psi(\mathbf{r})|^{4}
\end{aligned}
$$

The last term is a constant and can be dropped out. We start by minimizing the second term that corresponds to a quadratic form:

$$
\begin{aligned}
& -\sum_{\mathbf{r}}|\Psi(\mathbf{r})|^{2}\left|\sum_{\alpha=1}^{M} \beta_{\alpha} \phi_{\alpha}(\mathbf{r})\right|^{2} \\
& =-\sum_{\gamma=1}^{M} \sum_{\alpha=1}^{M}\left(\sum_{\mathbf{r}} \phi_{\gamma}(\mathbf{r})|\Psi(\mathbf{r})|^{2} \phi_{\alpha}(\mathbf{r})\right) \beta_{\gamma} \beta_{\alpha}
\end{aligned}
$$

Minimizing this quadratic form under the constraint $\|\beta\|=$ 1 can be done exactly by taking the eigenvector associated to the largest negative eigenvalue. This gives us a starting value of $\beta$ from which we minimize $L(\beta)$ through an iterative procedure. We define $\tilde{\Psi}(\mathbf{r})=\sqrt{|\Psi(\mathbf{r})|^{2}} \times \operatorname{sign}\left[\sum_{\alpha} \beta_{\alpha} \phi_{\alpha}(\mathbf{r})\right]$ and look for $\beta$ that minimizes the least square problem $\mid \tilde{\Psi}(\mathbf{r})-$ $\left.\sum_{\alpha} \beta_{\alpha} \phi_{\alpha}(\mathbf{r})\right|^{2}$. We iterate the procedure until the vector $\beta$ converges to a fixed point. We run this minimization procedure for all the sets of $M$ basis modes with adjacent energies and keep the one that gives the overall smallest $L(\beta)$. We then deduce $\Psi(\mathbf{r})$ as

$$
\Psi(\mathbf{r})=\operatorname{sign}\left[\sum_{\alpha=1}^{M} \beta_{\alpha} \phi_{\alpha}(\mathbf{r})\right] \sqrt{|\Psi(\mathbf{r})|^{2}}
$$

We have tested the procedure for different values of $M$ and observe that the deduced sign does not change for $M \geqslant 3$ (see Fig. S2 of the Supplemental Material [28]).

\section{APPENDIX C: OVERLAP AND HOPPING RATES FOR THE SI AND SII LATTICES}

From the numerical simulations of the charge and current distributions, we obtain the following overlap integrals

$$
\begin{aligned}
\left\langle E_{\mu} E_{v}\right\rangle_{0} & =\left(\begin{array}{cc}
\omega_{A} & 8.2 \times 10^{-3} \bar{\omega}_{A B} \\
8.2 \times 10^{-3} \bar{\omega}_{A B} & \omega_{B}
\end{array}\right) \\
\left\langle E_{\mu} E_{\nu}\right\rangle_{\mathbf{a}_{1}} & =\left(\begin{array}{cc}
2.2 \times 10^{-4} \omega_{A} & -1.9 \times 10^{-6} \bar{\omega}_{A B} \\
6.7 \times 10^{-3} \bar{\omega}_{A B} & 2.3 \times 10^{-4} \omega_{B}
\end{array}\right) \\
\left\langle E_{\mu} E_{\nu}\right\rangle_{\mathbf{a}_{2}} & =\left(\begin{array}{cc}
2.1 \times 10^{-4} \omega_{A} & -1.2 \times 10^{-5} \bar{\omega}_{A B} \\
1.3 \times 10^{-2} \bar{\omega}_{A B} & 2.2 \times 10^{-4} \omega_{B}
\end{array}\right) \\
\left\langle E_{\mu} E_{\nu}\right\rangle_{\mathbf{a}_{1}-\mathbf{a}_{2}} & =\left(\begin{array}{cc}
1.4 \times 10^{-4} \omega_{A} & 1.3 \times 10^{-4} \bar{\omega}_{A B} \\
1.0 \times 10^{-5} \bar{\omega}_{A B} & 1.4 \times 10^{-4} \omega_{B}
\end{array}\right) \\
\left\langle H_{\mu} H_{\nu}\right\rangle_{0} & =\left(\begin{array}{cc}
\omega_{A} & -3.1 \times 10^{-2} \bar{\omega}_{A B} \\
-3.1 \times 10^{-2} \bar{\omega}_{A B} & \omega_{B}
\end{array}\right) \\
\left\langle H_{\mu} H_{\nu}\right\rangle_{\mathbf{a}_{1}} & =\left(\begin{array}{cc}
-4.2 \times 10^{-3} \omega_{A} & -8.3 \times 10^{-4} \bar{\omega}_{A B} \\
-3.2 \times 10^{-2} \bar{\omega}_{A B} & -4.2 \times 10^{-3} \omega_{B}
\end{array}\right) \\
\left\langle H_{\mu} H_{\nu}\right\rangle_{\mathbf{a}_{2}} & =\left(\begin{array}{cc}
-4.2 \times 10^{-3} \omega_{A} & -8.4 \times 10^{-4} \bar{\omega}_{A B} \\
-2.8 \times 10^{-2} \bar{\omega}_{A B} & -4.2 \times 10^{-3} \omega_{B}
\end{array}\right) \\
\left\langle H_{\mu} H_{\nu}\right\rangle_{\mathbf{a}_{1}-\mathbf{a}_{2}} & =\left(\begin{array}{cc}
-4.2 \times 10^{-3} \omega_{A} & -2.5 \times 10^{-3} \bar{\omega}_{A B} \\
-2.6 \times 10^{-3} \bar{\omega}_{A B} & -4.2 \times 10^{-3} \omega_{B}
\end{array}\right)
\end{aligned}
$$

where $\bar{\omega}_{A B}=\sqrt{\omega_{A} \omega_{B}}$ and the $\mu$ and $v$ indices correspond respectively to the line and column indices of the matrix. Using $\omega_{A}=2 \pi \times 6.02 \mathrm{GHz}$ and $\omega_{B}=2 \pi \times 6.13 \mathrm{GHz}$, we 
obtain the following coupling matrices using Eq. (14):

$$
\begin{aligned}
J(0) & =2 \pi \times\left(\begin{array}{cc}
12 & 123 \\
123 & 12
\end{array}\right) \mathrm{MHz} \\
J\left(\mathbf{a}_{1}\right) & =2 \pi \times\left(\begin{array}{cc}
18 & 3.7 \\
121 & 18
\end{array}\right) \mathrm{MHz}
\end{aligned}
$$

$$
\begin{array}{r}
J\left(\mathbf{a}_{2}\right)=2 \pi \times\left(\begin{array}{cc}
18 & 3.8 \\
128 & 18
\end{array}\right) \mathrm{MHz} \\
J\left(\mathbf{a}_{1}-\mathbf{a}_{2}\right)=2 \pi \times\left(\begin{array}{cc}
18 & 10 \\
10 & 18
\end{array}\right) \mathrm{MHz} .
\end{array}
$$

[1] J. Koch and K. Le Hur, Superfluid-Mott-insulator transition of light in the Jaynes-Cummings lattice, Phys. Rev. A 80, 023811 (2009).

[2] J. Koch, A. A. Houck, K. L. Hur, and S. M. Girvin, Time-reversal-symmetry breaking in circuit-qed-based photon lattices, Phys. Rev. A 82, 043811 (2010).

[3] S. Schmidt and J. Koch, Circuit QED lattices: Towards quantum simulation with superconducting circuits: Circuit QED lattices, Ann. Phys. (NY) 525, 395 (2013).

[4] P. Roushan, C. Neill, A. Megrant, Y. Chen, R. Babbush, R. Barends, B. Campbell, Z. Chen, B. Chiaro, A. Dunsworth et al., Chiral ground-state currents of interacting photons in a synthetic magnetic field, Nat. Phys. 13, 146 (2017).

[5] M. Fitzpatrick, N. M. Sundaresan, A. C. Y. Li, J. Koch, and A. A. Houck, Observation of a Dissipative Phase Transition in a One-Dimensional Circuit QED Lattice, Phys. Rev. X 7, 011016 (2017).

[6] R. Ma, B. Saxberg, C. Owens, N. Leung, Y. Lu, J. Simon, and D. I. Schuster, A dissipatively stabilized Mott insulator of photons, Nature (London) 566, 51 (2019).

[7] Q. Guo, C. Cheng, H. Li, S. Xu, P. Zhang, Z. Wang, C. Song, W. Liu, W. Ren, H. Dong, R. Mondaini, and H. Wang, Stark ManyBody Localization on a Superconducting Quantum Processor, Phys. Rev. Lett. 127, 240502 (2021).

[8] G. P. Fedorov, S. V. Remizov, D. S. Shapiro, W. V. Pogosov, E. Egorova, I. Tsitsilin, M. Andronik, A. A. Dobronosova, I. A. Rodionov, O. V. Astafiev, and A. V. Ustinov, Photon Transport in a Bose-Hubbard Chain of Superconducting Artificial Atoms, Phys. Rev. Lett. 126, 180503 (2021).

[9] D. L. Underwood, W. E. Shanks, A. C. Y. Li, L. Ateshian, J. Koch, and A. A. Houck, Imaging Photon Lattice States by Scanning Defect Microscopy, Phys. Rev. X 6, 021044 (2016).

[10] A. J. Kollár, M. Fitzpatrick, and A. A. Houck, Hyperbolic lattices in circuit quantum electrodynamics, Nature (London) 571, 45 (2019).

[11] L. Lu, J. D. Joannopoulos, and M. Soljačić, Topological photonics, Nat. Photonics 8, 821 (2014).

[12] A. B. Khanikaev and G. Shvets, Two-dimensional topological photonics, Nat. Photonics 11, 763 (2017).

[13] T. Ozawa, H. M. Price, A. Amo, N. Goldman, M. Hafezi, L. Lu, M. C. Rechtsman, D. Schuster, J. Simon, O. Zilberberg, and I. Carusotto, Topological photonics, Rev. Mod. Phys. 91, 015006 (2019).

[14] J. C. Culbertson, H. S. Newman, and C. Wilker, Optical probe of microwave current distributions in high temperature superconducting transmission lines, J. Appl. Phys. 84, 2768 (1998).

[15] A. P. Zhuravel, C. Kurter, A. V. Ustinov, and S. M. Anlage, Unconventional $\mathrm{rf}$ photoresponse from a superconducting spiral resonator, Phys. Rev. B 85, 134535 (2012).

[16] A. S. Averkin, A. P. Zhuravel, A. Karpov, S. M. Anlage, and A. V. Ustinov, Ultra-compact superconductive resonator with double-spiral structure, in 2013 7th International Congress on Advanced Electromagnetic Materials in Microwaves and Optics (IEEE, Talence, France, 2013), pp. 142-144.

[17] H. Wang, A. P. Zhuravel, S. Indrajeet, B. G. Taketani, M. D. Hutchings, Y. Hao, F. Rouxinol, F. K. Wilhelm, M. D. LaHaye, A. V. Ustinov, and B. L. T. Plourde, Mode Structure in Superconducting Metamaterial Transmission-Line Resonators, Phys. Rev. Applied 11, 054062 (2019).

[18] W.-P. Huang, Coupled-mode theory for optical waveguides: An overview, J. Opt. Soc. Am. A 11, 963 (1994).

[19] Sonnet ${ }^{\mathrm{TM}}$ ver. 15.52, Sonnet Software Inc., North Syracuse, NY, (2014).

[20] D. A. Powell, M. Lapine, M. V. Gorkunov, I. V. Shadrivov, and Y. S. Kivshar, Metamaterial tuning by manipulation of near-field interaction, Phys. Rev. B 82, 155128 (2010).

[21] V. Lomanets, O. Zhuromskyy, G. Onishchukov, and U. Peschel, Electrical circuit model of arrays of resonant elements, Phys. Rev. B 85, 125110 (2012).

[22] S. Y. Elnaggar, R. J. Tervo, and S. M. Mattar, Energy coupled mode theory for electromagnetic resonators, IEEE Trans. Microwave Theory Tech. 63, 2115 (2015).

[23] G. W. Semenoff, Condensed-Matter Simulation of a ThreeDimensional Anomaly, Phys. Rev. Lett. 53, 2449 (1984).

[24] A. Morvan, M. Féchant, G. Aiello, J. Gabelli, and J. Estève, Observation of topological valley Hall edge states in honeycomb lattices of superconducting microwave resonators, Opt. Mater. Express 11, 1224 (2021).

[25] A. P. Zhuravel, A. G. Sivakov, O. G. Turutanov, A. N Omelyanchouk, S. M. Anlage, A. Lukashenko, A. V. Ustinov, and D. Abraimov, Laser scanning microscopy of HTS films and devices (Review Article), Low Temp. Phys. 32, 592 (2006).

[26] J. Aiken, J. A. Erdos, and J. Goldstein, On löwdin orthogonalization, Int. J. Quantum Chem. 18, 1101 (1980).

[27] J. D. Jackson, Classical Electrodynamics, 2nd ed. (Wiley, New York, 1975).

[28] See Supplemental Material at http://link.aps.org/supplemental/ 10.1103/PhysRevResearch.4.013085 for details on the microwave setup and illustration of the sign reconstruction on one specific mode. 